\title{
17TH ASTIN COLLOQUIUM, LINDAU (WEST GERMANY), 2-6 OCTOBER 1983
}

The scientific part of the Lindau meeting was made up by four working sessions, one of them reserved for the traditional "Speaker's Corner" and the other three for the main subjects of the Congress:

Subject 1: The influence of different risk sharing arrangements on the risk behaviour of the participants in the direct insurance and reinsurance markets. Subject 2: Data problems, statistical methods and numerical procedures in non-life insurance.

Subject 3: Planning and forecasting the technical and non-technical results of an insurance company.

Planning and forecasting - the subject of the first working session-are certainly well established in the daily practice of insurance companies. Regarding the papers submitted to the Colloquium, it is interesting to see that most of them deal only with pure mathematical forecasting especially of technical results and necessary technical reserves. This indicates that actuaries are not so much involved in the planning process as a whole and in the forecasting of non-technical results of an insurance company. Only the paper by STRAUB deals with the question "what can the actuary do in corporate planning?" He gives several examples out of his practical experience in a reinsurance company and considers the special case of the so-called "Cat Fund", i.e. the determination of the necessary risk capital for limiting the risk of a portfolio in a reasonable way.

Other tasks which actuaries can tackle in the planning process are:

- the breakdown of overall risk capital into subportfolios;

- improving scarce statistical material by simulation methods;

- comparing actual to planned figures (judging the "credibility" of the profit centre planning);

- quantifying the change in IBNR.

This latter problem of estimating the claims reserves is certainly one of the most prominent actuarial problems, of today. In this working session, the subject "claims reserves" was dealt with in the papers by DE FERRA, SöDERSTRÖM and HERTIG. In the paper by DE FERRA an idea of Hachemeister has been taken up to describe the evolution of a claim by a Markov stochastic process. From a theoretical point of view, this model is very appealing and first empirical tests have shown a remarkable stability of the transition probabilities. The approach will certainly be pursued.

The paper by SöDERSTRÖM gives some practical calculation methods for the determination of reserves in group sickness insurance. It should be mentioned, however, that in this class of business with its extensive population and its homogeneous claims data estimation of reserves is relatively easy. The interesting paper by HERTIG deals with the estimation of reserves in marine reinsurance. 
He uses a lognormal distribution for the logarithmic increments of the loss ratios of consecutive years of development. This paper is a further indication that more and more reserving methods giving confidence intervals for the estimation of the necessary reserve are used, the statistical model assumptions of which can be tested.

Looking at the different purposes for which forecasts are made in insurance business, one of the most important is the determination of solvency reserves. At the colloquium NorberG and SuNDT reported on a proposed system for solvency control at present discussed in Norway. They emphasized the following aspects of solvency control:

- sufficiency of the technical reserves;

- rules for the valuation of the assets;

- regular control;

- priority to insurance claims in case of bankruptcy;

- unified system for the reporting of statistical data.

The paper gave rise to an interesting discussion on the different aspects of solvency control, the impact of fluctuations in the non-technical results, business cycles and the role of the supervisory authorities. Business cycles were also the subject of a short paper by BoHMAN which was included in the discussion. A sophisticated forecasting model concerning premium rating formulas was presented in the paper by RANTALA where the framework of Kalman-filter-techniques was used to derive premium rating formulas which minimize premium fluctuations under given constraints on the solvency margin. Concluding remark to this working session could be the statement that there is still a long way to go before the non-technical aspects of insurance will be incorporated in actuarial methods in such a way that they are helpful to solve practical problems of insurance economics.

The second working session of the Congress discussed Subject 1 "The influence of different risk sharing arrangements on the risk behaviour of the participants in the direct insurance and reinsurance markets."

Risk sharing arrangements are the daily practice of insurance and reinsurance and there are important questions to be answered in this context, for example:

Which forms of risk sharing are to be chosen?

What should be the retention of each party?

How should risk premiums (loss expectancy and risk loading) be calculated?

Recently, particular progress has been made in the calculation of the loss expectancy (recursive algorithms, stop-loss premiums) and a number of premium principles for calculating the risk loading has been proposed. Although quite a number of actuarial theorems is known concerning risk sharing arrangements (the results on the optimality of different risk sharing arrangements by Borch, Bühlmann, Arrow et al. under special assumptions should be mentioned), the results of risk theory in this field have still been unsatisfactory under a practical point of view: 
- premium calculation models are unrealistic as they do not include investment income and administration costs;

- there is a considerable amount of uncertainty in estimating the loss distributions ignored in the models of risk sharing;

- there exist accumulations of risk by the risk-accepting party;

- aspects like negotiation power are neglected;

- mostly risk-sharing is only regarded for one period;

- the choice of the suitable optimality criterion is still open and not fully discussed.

Some of these shortcomings of the existing models had been incorporated in the more detailed description of this subject in the hope that investigations into more realistic models would be carried out.

It has to be said that the papers presented under Subject 1 do not deal so much with these shortcomings from a practical point of view as with aspects of the three questions asked at the beginning.

Three papers deal with the most important form of non-proportional risk sharing, the deductible. In the paper "A note on an aspect of dangerousness of deductibles ..." ALBRECHT criticizes the application of the coefficient of variation as a measure of risk.

He proposes the evaluation of risk by methods of utility theory. This led to a controversy stated in the paper by MACK and in a second paper by ALBRECHT. The discussion which followed the presentation of these papers can be summarized in the way that there is only a contradiction between the evaluation by utility theory and by the coefficient of variation when they are used as a measure for the same definition of dangerousness of claims distributions.

The paper by BORCH discusses the question how the safety loading has to be calculated. Since none of the numerous premium principles developed has found general acceptance, he attempts to clarify whether under certain market conditions rational behaviour may lead to a premium principle that is valid for all insurance companies. While BORCH regards the situation of a symmetrical risk exchange pool, the model of GERBER examines the situation where a portfolio is passed on from one insurer to exactly one reinsurer and so on. A hierarchical chain of companies thus shares the risk whereby only proportional risk sharing is regarded. The amount ceded and the loading factor in the premium are determined by a bargaining process. The results are very informative and helpful for the further investigation of this risk sharing problem.

While Gerber's paper deals with optimality investigations for forms of proportional risk sharing, in practice there are often non-proportional forms of risk sharing for which even the calculation of expected claims causes great difficulties: These problems are the topic of the papers by KREMER and MACK. KREMER discusses the largest claim reinsurance and its generalizations. The special significance of his paper lies in its theoretical content as the results important for practical forms of reinsurance were already given in a former paper by Kremer.

Under various assumptions on the claims distribution, especially looking at the case of a log-normal distribution, MACK treats the case where in addition to the 
deductible an annual limit on the aggregate loss exists. He examines the influence of changes in the model parameters and arrives at rating curves which are useful for the underwriting practice.

A quite different approach to the question of risk-sharing is taken by HELTEN and BECK. They have tried to analyse the present risk-sharing behaviour of German direct insurers by means of a questionnaire. In their paper, they report on the answers given in respect of the objectives pursued by the companies when taking reinsurance.

Subject 2 was entitled "Data problems, statistical methods and numerical procedures" and its heterogeneity was reflected by the papers presented. It is especially noteworthy to mention that quite a number of papers analysed empirical data with rather advanced statistical methods. A rough classification can be achieved by grouping them into "Statistical methods and statistical analysis of empirical data" and "Numerical procedures". To the first group belong papers on the analysis of claim numbers, the analysis of motor insurance problems and on the analysis of fire claims.

In the paper by ALBRECHT "Credibility for claim numbers ..." an evolutionary credibility model (the underlying risk parameter changes in time) for the successive claim numbers of a single risk is examined. Its central result is that-in the case of the sequence of risk parameters being an arbitrary weakly stationary process-it is possible to calculate the coefficients of the credibility estimator (not the estimator itself!) recursively, as well as the mean square error. The paper examines the problem of estimating the structural parameters from collective data and considers various special cases.

In their paper on the analysis of claim numbers, AJNE and ANDERSSON use a particular ARIMA-model to forecast future claim numbers. The basic data consist of 84 monthly claim number figures (1975-1981) of householders comprehensive and of motor hull insurance. The authors report on some performed forecastings and their a posteriori comparison of estimated and true values indicates a reasonable performance.

In his paper on motor premium rating, CouTTs deals with nearly every aspect relevant to motor premium rating (forecasting, constructing tariff classes, expense allocation, marketing aspects, surplus analysis). Much emphasis is given to the treatment of practical problems arising when analysing company portfolios, a problem of special importance in countries where the tariff structure is not determined by supervisory authorities or insurance associations. In his paper, Alting von GeUSAU describes a model for analysing the effects of different bonus-malus-systems. He uses data from the Italian BM-system to demonstrate the usefulness of his model for answering different questions on premium development in time, i.e. to investigate whether the premium income remains adequate while the insured move to higher bonus classes.

The paper by RAMLAU-HANSEN reports on an empirical analysis of fire claims for single family houses from a major Danish non-life insurance company. As individual claim amount distribution a log-gamma distribution with a Pareto-type tail is used. In addition, a kind of graduation is performed by assuming that the 
expected claim amount is linearly increasing with the size of the house. The claim number distribution used is a Poisson distribution with a particular form of the parameter taking into account policy years and reporting time. Results on the linear dependence of the net risk premium on the size of the house, on the standard deviation of the total claim amount, on the skewness of the distribution and on the necessary risk loading are given, which demonstrate very clearly the big risk of fire portfolios.

During the last years, risk theory has made considerable progress in the determination of the claims distribution by numerical procedures through the application of recursive methods. A computing method has been developed by BERTRAM using properties of characteristic functions and the tool of the fast Fourier transform for numerical performance. While the recursive methods have considerable problems with negative risk sums (a case relevant for pension insurance), BERTRAM's method exhibits no such problems and, in addition, consumes very little computer time. This effect will be especially useful, if the Poisson intensity is large.

The paper by ETrL proves an interesting theoretical feature in this context. Starting from the well-known relation between the Laplace transform of the claim amount distribution and the aggregate claim distribution, he arrives at an integral equation for the accumulated claims distribution, a discretization of which leads to a recursive formula. Interesting theoretical results are also derived in the paper by NETZEL where the influence of different factors on the probability of ruin for an infinite time horizon is investigated. An integro-differential equation for the general problem is presented and in addition a closed expression is obtained for the case of an exponential distribution and an infinite retention. The probability of ruin is also dealt with in the paper by GoOvAERTS and DE VYLDER. They develop a stable (there exists a bound for the rounding errors) recursive algorithm for the calculation of the probability of ruin for an infinite time horizon and a fixed initial capital. The approximation error for the true ruin probability can be made arbitrarily small.

Summarizing Subject 2 it can be said that not only risk theory has been developed further in the last years, also the application of risk theoretical models to practical problems has made significant progress.

Last, but not least there were quite a lot of different papers presented at "Speaker's corner" during the colloquium. These range from papers of more theoretical interest discussing extension of risk theoretical models like ALBRECHT "Laplace transform ...", JANSSEN/REINHARD and REICH to papers discussing practical problems like premium calculation for bank robbery and spoliation insurance (PEREZ). Of particular interest are the papers by LEMAIRE dealing with the problem of the cost loading included in a commercial premium rate. He demonstrates that the proportional loading-mostly used in practice-will often result in an unfair allocation of the expenses to the different risks. He shows that the problem of cost allocation can be dealt with in a game-theoretical framework. The problems of determining a reasonable cost allocation is equivalent to determining an imputation of the core of a cooperative $N$-person game. This 
correspondence allows to apply results of game theory to derive special cost allocations. Lemaire shows that only one of the allocation methods regarded by him satisfies a set of reasonable postulates.

Besides the working sessions there were two lectures held during the colloquium. The first was by Professor DANNER on "the structure of risk classification in motor own damage insurance and its influence on motor car construction". Professor DANNer was engaged in the construction of a tariff for motor own damage insurance in Germany and reported on the tariff class construction depending on the repair costs of the specific models and on the claims frequency caused by the drivers of these models. In particular he pointed out that this tariff caused car manufacturers to put more emphasis on lower repair costs when constructing new models.

The second lecture was given by Professor FeILMEIER on "Numerical methods in calculating the aggregate loss distribution". He summarized the significant progress made in this field during the last years and commented in particular on the recursive methods and on the method using the fast Fourier transform. He underlined that the problem of numerical calculation of the aggregate claim distribution should no longer prevent anyone from using risk theoretical models in practice. This summary seems to be typical for the whole colloquium as most speakers emphasized the necessity of incorporating the well-developed risktheoretical methods into the solution of practical problems, a classical concern of Astin.

$$
\text { P. Albrecht, K. Flemming, E. Kremer and T. Mack }
$$

\section{Subject 1: The influence of different risk-sharing arrangements on the risk behaviour of the participants of the direct and reinsurance markets}

$P$. Albrecht, A note on an "aspect of dangerousness" of deductibles-a criticism of the coefficient of variation.

Increasing risk and deductibles.

$K$. Borch, Equilibrium premiums in an insurance market.

H.U. Gerber, Chains of reinsurance.

E. Helten and D. Beck, Optimal reinsurance-a scientific fiction?

E. Kremer, An asymptotic formula for the net premium of some reinsurance treaties.

T. Mack, Premium calculation for deductible policies with an aggregate limit. The utility of deductibles from the insurer's point of view.

Subject 2: Data problems, statistical methods and numerical procedures in non-life insurance

B. Ajne and $K$. Andersson, A note on time series analysis of numbers of claims. $P$. Albrecht, Credibility for claim numbers in the case of a time dependent structure function: an application of doubly stochastic Poisson sequencies.

B. Alting von Geusau, The matrix method to solve motor insurance problems.

J. Bertram, Calculation of aggregate claims distributions in case of negative risk sums. 
S. Coutts, Motor premium rating.

W. Ettl, Recursive formulas for compound distributions by Laplace transformation methods.

M. Goovaerts and F. De Vylder, A stable recursive algorithm for evaluation of ultimate ruin probabilities.

C. Netzel, Numerical study concerning ruin probability.

H. Ramlau-Hansen, Fire claims for single family houses.

Subject 3: Planning and forecasting technical and non-technical results of an insurance company

H. Bohman, Business cycles.

C. de Ferra, A stochastic model for the analysis and evaluation of the claims reserve.

$J$. Hertig, A statistical approach to IBNR-reserves in marine reinsurance.

$N$. E. Masterson, Non-life insurance short term forecasting.

R. Norberg and B. Sundt, Draft of a system for solvency control in non-life insurance.

$J$. Rantala, Experience rating of claims processes with stochastic trends.

L. G. Söderström, A practical application of an IBNR process for an almost stationary business.

E. Straub, Actuarial remarks on planning and controlling in reinsurance.

\section{Speaker's Corner}

P. Albrecht, Laplace transforms, Mellin transforms and mixed Poisson processes.

J. Janssen and J. M. Reinhard, Formes explicites de probabilités de ruine pour une classe de modèles de risque semi-markoviens.

$W$. S. Jewell and $R$. Schnieper, Credibility theory for second moments.

$J$. Lemaire, An application of game theory: cost allocation.

The influence of expense loadings on the fairness of a tariff.

A. Martinez Vazquez, Le test d'adherence des fonctions de repartition à type discrete dans l'assurance non-vie.

E. Prieto Pérez, Analysis of bank robbery and spoliation insurance.

A. Reich, Premium principles and translation invariance. 